\title{
Erratum: Generalized quantum process discrimination problems [Phys. Rev. A 103, 062606 (2021)]
}

\author{
Kenji Nakahira $\odot$ and Kentaro Kato
}

(Received 22 November 2021; published 3 December 2021)

DOI: 10.1103/PhysRevA.104.069902

Page 4, the second line of Eq. (5) should read " $C_{\mathrm{G}}:=\operatorname{Pos}_{\tilde{V}}^{M} . "$

Page 11, Example 13, the last sentence (which starts with "Thus, it is easily seen") should read "Thus, it is easily seen from Example 11 that this problem is $\mathcal{H}^{T}$ symmetric if $\Phi^{(g)}$ belongs to $C_{\mathrm{G}}$ for any $\Phi \in C_{\mathrm{G}}$."

Page 17, Sec. VI A 1, the second sentence of the first paragraph (which starts with "We recall that") should read "Assume that $\Phi^{(g)} \in C_{\mathrm{G}}\left(\forall \Phi \in C_{\mathrm{G}}\right)$ holds; then, this problem is $\mathcal{H}^{T}$ symmetric."

Page 17, Sec. VI A 1, the third and fourth sentences of the second paragraph (which starts with "Assume that a collection of channels") should read "Assume that a collection of channels $\left\{\hat{\Lambda}_{r}\right\}_{r=0}^{R-1}$ is teleportation covariant, i.e., there exists a projective unitary representation $h \mapsto U_{h}$ such that

$$
\hat{\Lambda}_{r}=\operatorname{Ad}_{U_{h}} \circ \hat{\Lambda}_{r} \circ \operatorname{Ad}_{\tilde{U}_{h}}, \quad \forall r \in \mathcal{I}_{R}, h \in \mathcal{H} .
$$

It is easily seen that $\Phi^{(g)} \in C_{\mathrm{G}} \quad\left(\forall \Phi \in C_{\mathrm{G}}\right)$ holds and $h \mapsto \tilde{U}_{h} \quad(h \in \mathcal{H})$ is irreducible, and thus there exists a tester with maximally entangled pure states that is optimal."

Page 17, Sec. VI A 1, the first sentence of the third paragraph (which starts with "The second example is") should read " The second example is the case in which $T=1$ holds and $\hat{\Lambda}_{0}, \ldots, \hat{\Lambda}_{R-1} \in \mathrm{Chn}(V, W)$ are unital qubit channels, i.e., unital channels with $N_{V}=N_{W}=2$."

Page 17, Sec. VI A 1, the last sentence of the third paragraph (which starts with "It follows that") should read "It follows that $\Phi^{(g)} \in C_{\mathrm{G}}\left(\forall \Phi \in C_{\mathrm{G}}\right)$ holds and the representation $h \mapsto U_{h}$ is irreducible, and thus there exists a tester with maximally entangled pure states that is optimal." 